\title{
Novel and Direct Access to the Human Locomotor Spinal Circuitry
}

\author{
Yury Gerasimenko, ${ }^{1,3}$ Ruslan Gorodnichev, ${ }^{2}$ Ekaterina Machueva, ${ }^{2}$ Elena Pivovarova, ${ }^{2}$ Denis Semyenov, ${ }^{2}$ \\ Alexandr Savochin, ${ }^{1}$ Roland R. Roy, ${ }^{3,5}$ and V. Reggie Edgerton ${ }^{3,4,5}$ \\ ${ }^{1}$ Pavlov Institute of Physiology, St. Petersburg 199034, Russia, ${ }^{2}$ Velikie Luky State Academy of Physical Education and Sport, Velikie Luky 182100, Russia, \\ and Departments of ${ }^{3}$ Physiological Science and ${ }^{4}$ Neurobiology and ${ }^{5}$ Brain Research Institute, University of California, Los Angeles, Los Angeles, California \\ 90095
}

The degree of automaticity of locomotion in primates compared with other mammals remains unclear. Here, we examine the possibility for activation of the spinal locomotor circuitry in noninjured humans by spinal electromagnetic stimulation (SEMS). SEMS (3 Hz and 1.3-1.82 tesla) at the T11-T12 vertebrae induced involuntary bilateral locomotor-like movements in the legs of individuals placed in a gravity-neutral position. The formation of locomotor-like activity during SEMS started with a latency of $0.68 \pm 0.1 \mathrm{~s}$ after delivering the first stimulus, unlike continuous vibration of muscles, which requires several seconds. The first EMG burst in response to SEMS was observed most often in a proximal flexor muscle. We speculate that SEMS directly activates the circuitry intrinsic to the spinal cord, as suggested by the immediate response and the electrophysiological observations demonstrating an absence of strictly time-linked responses within the EMG burst associated with individual stimuli during SEMS. SEMS in the presence of vibration of the leg muscles was more effective in facilitating locomotor-like activity than SEMS alone. The present results suggest that SEMS could be an effective noninvasive clinical tool to determine the potential of an individual to recover locomotion after a spinal cord injury, as well as being an effective rehabilitation tool itself.

\section{Introduction}

The ability to generate actual, as opposed to fictive, locomotion in the absence of brain input can be attributed to the combination of the circuitry intrinsic to the lumbosacral spinal cord, i.e., central pattern generation, and the ability of this circuitry to process complex proprioceptive and cutaneous patterns associated with stepping. Thus, an important clinical issue is how to gain access to and capitalize on this highly integrated sensorimotor circuitry in facilitating recovery of locomotion after neuromotor disorders resulting in deficits in locomotor ability.

Two strategies that can facilitate stepping movements are as follows: (1) continuous vibration of the quadriceps and hamstring muscle groups, and (2) continuous stimulation of the peroneal or sural nerve. A tonic presentation of these relatively nonspecific stimuli can generate cyclic locomotor-like movements of the lower limbs of some noninjured individuals when the legs are supported in a gravity-neutral position (Gurfinkel et al., 1998; Selionov et al., 2009). These two strategies may reflect the activation of similar functional lumbosacral circuitries in response to tonic epidural stimulation at approximately L2 in sub-

Received Sept. 23, 2009; revised Jan. 11, 2010; accepted Jan. 29, 2010.

This study was supported by National Institute of Neurological Disorders and Stroke Grant NS16333, Russian Foundation for Basic Research and U.S. Civilian Research and Development Foundation Grant RUB1-2872-ST-07, Russian Foundation for Basic Research Grant 07-04-00526, the Christopher and Dana Reeve Foundation, and the California Roman Reed Bill.

Correspondence should be addressed to Dr. V. Reggie Edgerton, Department of Physiological Science, at the above address. E-mail:vre@ucla.edu.

DOI:10.1523/JNEUROSCI.4751-09.2010

Copyright $\odot 2010$ the authors $\quad 0270-6474 / 10 / 303700-09 \$ 15.00 / 0$ jects with a clinically defined complete spinal cord injury (Dimitrijevic et al., 1998; Minassian et al., 2007; Gerasimenko et al., 2008), in complete spinal rats when epidurally stimulating lumbosacral segments (Ichiyama et al., 2005; Gerasimenko et al., 2007; Lavrov et al., 2008; Courtine et al., 2009), and in acute decerebrated cats when epidurally stimulating at L5 (Gerasimenko et al., 2009a).

Given the extensive experience in humans with electromagnetic stimulation (Hallett, 2000, 2007; Di Lazzaro et al., 2004; Fitzgerald et al., 2006; Huerta and Volpe, 2009), we examined the feasibility of using spinal electromagnetic stimulation (SEMS) to induce locomotor-like movements in noninjured individuals. In the present paper we describe robust, locomotor-like movements of the lower limbs in response to tonic SEMS when the subject's legs were placed in a gravityneutral position.

Parts of this paper (preliminary results) have been published previously (Gerasimenko et al., 2009b).

\section{Materials and Methods}

Experimental subjects

Sixty-five individuals (students of Velikie Luky State Academy of Physical Education and Sport, Velikie Luki, Russia) participated in the study. The subject pool was of mixed gender and had a mean age of 24 years. All subjects signed voluntary written consent forms to participate in these experiments. The study was approved by the Human Subject Protection Committee at the Velikie Luky State Academy of Physical Education and Sport, Velikie Luki, and conformed to the principles stated in the Declaration of Helsinki. 


\section{Experimental procedures}

The experimental setup was similar to that described previously (Gurfinkel et al., 2000; Selionov et al., 2009). We used a two-legged suspension system. Briefly, the subject laid on the left side with the right (upper) leg supported directly in the area of the shank and the left (lower) leg placed on a rotating brace attached to a horizontal board $(1155 \times 200 \mathrm{~mm}$, weight $3 \mathrm{~kg}$ ) supported by vertical ropes secured to hooks in the ceiling (supplemental videos 1-4, available at www.jneurosci.org as supplemental material). In this position the subjects could perform voluntary air stepping movements with large amplitudes. The subjects were instructed not to voluntarily intervene with the movements induced by SEMS. During each recording session the subjects in the placebo control condition heard the same sound of the SEMS as if they were being simulated, but no SEMS was applied. No leg movements were observed in any of the subjects in the placebo control condition.

In the initial two or three experimental sessions for each subject the focus was on defining the most optimal stimulation parameters to elicit stepping by using frequencies of $1,3,5,10$, and $20 \mathrm{~Hz}$. During the same session we also tested the responses at strengths of $40,50,60$, and $70 \%$ of maximum at $3 \mathrm{~Hz}$. A consistent observation was that a frequency of $3 \mathrm{~Hz}$ at a strength of $60 \%$ maximal was optimal for inducing stepping. Based largely on these stimulation parameters we also stimulated at different vertebral levels, specifically between $\mathrm{T} 11$ and $\mathrm{T} 12$, T12 and L1, L1 and L2, and L2 and L3. In all cases the most effective site for stimulation was between vertebral levels T11 and T12. After having defined these stimulation parameters, we then tested all seven subjects with the same protocol. This protocol consisted of stimulation at $1,3,5,10$, and $20 \mathrm{~Hz}$ at $60 \%$ maximum strength. In addition, we tested the effects of the strength of stimulation at $40,50,60$, and $70 \%$ of maximum at $3 \mathrm{~Hz}$. While similar results were obtained at 3 and $5 \mathrm{~Hz}, 3 \mathrm{~Hz}$ stimulation elicited stepping and allowed for longer stimulation periods without overheating the stimulation unit. In light of this, the lower stimulation frequency was selected to perform the standardized testing in the final session. Thus, the experimental variables tested in the final session in all seven subjects was stimulation at $40,50,60$, and $70 \%$ maximum strength at $3 \mathrm{~Hz}$ between vertebral levels T11 and T12 followed by $1,3,5,10$, and $20 \mathrm{~Hz}$ at $60 \%$ maximum (see supplemental videos 1-4, available at www.jneurosci.org as supplemental material). In addition, stimulation at $3 \mathrm{~Hz}$ and $60 \%$ maximum was applied between vertebral levels T12 and L1, L1 and L2, and L2 and L3. Immediately following these tests each subject was stimulated with vibration for $30 \mathrm{~s}$, allowing $\sim 2$ min between the next session, which consisted of $10 \mathrm{~s}$ of vibration followed by $10 \mathrm{~s}$ of vibration plus SEMS ( $3 \mathrm{~Hz}$ at $60 \%$ maximum between vertebral levels T11 and T12). As a final test, each subject was asked to generate a stepping-like motion voluntarily for $20 \mathrm{~s}$. All test sessions were completed within a $2 \mathrm{~h}$ period. It was during this final test session that all group mean data were obtained.

\section{EMG recording and movement analyses}

Bipolar surface electrodes were placed bilaterally on rectus femoris (RF) and biceps femoris (BF). The electrodes were positioned on the muscle belly, midway between the origin and insertion of the RF and BF as described previously (Courtine et al., 2007). EMG signals were differentially amplified (bandwidth of $10 \mathrm{~Hz}$ to $10 \mathrm{kHz}$ ) and digitized at $2 \mathrm{kHz}$ A-M Systems, model 1700). To avoid artifacts from the SEMS, the EMG signals were passed through a low-pass filter with a cutoff frequency of $100 \mathrm{~Hz}$. The filtered EMG signals were analyzed off-line to compute the amplitude, duration, and timing of individual bursts. The latency of locomotor-like activity was calculated from the initiation of stimulation (SEMS, vibration, or SEMS during vibration) to the first EMG burst in any leg muscle.

Movements of the right (upper) leg were monitored using a digital video camera placed perpendicular to the leg. Reflective markers were placed on the lateral epicondyle of the humerus, greater trochanter, lateral epicondyle of the femur, lateral malleolus, and hallux. Angular movements of the hip joint were derived from the markers on the lateral epicondyle of the humerus, greater trochanter, and lateral epicondyle of the femur. Knee movements were derived from the markers placed at the greater trochanter, lateral epicondyle of the femur, and lateral malleolus.
Ankle movements were derived from markers at the lateral epicondyle of the femur, lateral malleolus, and hallux. Markers at the greater trochanter, lateral epicondyle of the humerus, lateral malleolus, and hallux were used to reconstruct the kinematics of the stepping movements. Motion capture software was used to obtain the coordinates of the markers. The video and EMG recordings were synchronized, and the mean cycle period and amplitudes of the hip, knee, and ankle displacements for 10-12 cycles were determined.

The cycle period during all stimulating paradigms was defined as the distance between two peaks of the extension excursion of the angular movement of the hip, knee, and ankle joints. The phase shift between the knee and hip movements was defined as the interval between the peaks of extension excursion at these joints. One step cycle during stable stepping is illustrated to show the coordination between joint movements. The total duration of the testing session was $\sim 2 \mathrm{~h}$. During subsequent testing sessions the order of the experimental conditions was randomized.

\section{Stimulation procedures}

Electromagnetic spinal cord stimulation. The magnetic impulses were generated by a Magstim Rapid ${ }^{2}$ stimulator. A circular coil $(70 \mathrm{~mm})$ was placed over the T11-T12, T12-L1, L1-L2, and L2-L3 vertebrae. One $10 \mathrm{~s}$ train of impulses at $1,3,5,10$, and $20 \mathrm{~Hz}$ was applied to the spine at a strength of $40 \%$ (1.04 tesla), 50\% (1.3 tesla), $60 \%$ (1.56 tesla), and 70\% (1.82 tesla) from the maximum output of the stimulator (2.6 tesla).

Vibration. The vibrators were aluminum cylinders $(33 \mathrm{~mm}$ diameter and $90 \mathrm{~mm}$ length) that enclosed a DC motor attached with eccentric weights on their two axes. Rubber belts were used to fix the vibrators over the distal tendon of the RF and BF bilaterally. The amplitude of the vibration was $\sim 1 \mathrm{~mm}$ and the frequency $\sim 50 \mathrm{~Hz}$. All four muscles were vibrated simultaneously during testing.

\section{Statistical procedures}

The values are reported as mean \pm SE. Overall significant differences among the variables studied during SEMS, vibration, SEMS plus vibration, and voluntary air stepping were determined using a two-way ANOVA. Significant group differences were determined using least significant difference post hoc analyses. Statistical significance was determined at $p<0.05$.

\section{Results}

Of the 65 subjects tested, $7(\sim 10 \%)$ demonstrated relatively robust locomotor-like activity in response to SEMS or vibration. This subject selectivity in locomotor responses could not be attributed to experimental variability, because every subject that demonstrated a response to SEMS or vibration during the first testing session demonstrated a similar response during every subsequent testing session. The implications of the selective nature of the generation of stepping-like activity are interesting and need to be examined further. For example, there could be different levels of supraspinally mediated inhibition of the spinal circuitry from subject to subject. There was a total of 23 testing sessions for the seven subjects studied. All of the quantitative group statistical comparisons were derived from the final test session for each subject (10-12 step-like cycles per experimental condition for seven subjects unless stated otherwise). There was no discomfort reported by any of the subjects in response to SEMS or vibration.

\section{SEMS-induced involuntary locomotor-like activity}

\section{Site of SEMS}

Figure 1 demonstrates the effectiveness of SEMS (3 Hz and 50\% maximum tesla) at different spinal segments for inducing involuntary locomotor-like movements in the legs of one individual placed in a gravity-neutral position. SEMS at T11-T12 vertebral level evoked rhythmic, involuntary, bilateral, locomotor-like movements, accompanied by corresponding rhythmic EMG activity in the leg muscles (Fig. $1 \mathrm{~A}$ ). SEMS at T12-L1 vertebral level induced low-amplitude leg movements and weak EMG activity 
(Fig. $1 B$ ). Stimulation at L1-L2 or L2-L3 vertebral level did not induce well defined, consistent locomotor-like movements in the legs and produced minimal EMG activity (Fig. 1C,D). Thus, the most effective locomotor movements were induced consistently with SEMS at T11-T12 vertebral level.

In our previous studies using epidural spinal cord stimulation, we reported that stimulation at the L2 spinal segment (T11T12 vertebral level) induced locomotor-like movements in the legs in spinal cordinjured patients more effectively than stimulation at more rostral or caudal segments (Dimitrijevic et al., 1998). In addition, percutaneous stimulation at the T11-T12 vertebral level was most effective in evoking multisegmental, monosynaptic responses in several leg muscles in normal individuals (Courtine et al., 2007).

Frequency of SEMS

The effect of frequency $(1,5,10$, and $20 \mathrm{~Hz}$ at optimal strength for each individual: $50-60 \%$ maximum tesla applied at the T11-T12 vertebral level) of SEMS on the kinematics and EMG characteristics of locomotor-like activity in the legs is shown in Fig. 2. SEMS at $1 \mathrm{~Hz}$ did not induce any clear, sustained locomotorlike activity in the leg muscles (Fig. 2A). Locomotor-like movements, however, were observed at 5, 10, and $20 \mathrm{~Hz}$. The latency (calculated from the initiation of SEMS to the first EMG burst in the BF) progressively decreased from $\sim 1.2 \mathrm{~s}$ at 5 $\mathrm{Hz}$ to $\sim 1.0 \mathrm{~s}$ at $10 \mathrm{~Hz}$ to $\sim 0.6 \mathrm{~s}$ at $20 \mathrm{~Hz}$.

At all frequencies the leg movements began with hip extension followed by knee flexion, with a phase shift between the hip and knee joint movements of $\sim 0.5, \sim 0.3$, and $\sim 0.2 \mathrm{~s}$ at 5,10 , and 20 $\mathrm{Hz}$, respectively (Fig. $2 \mathrm{~A}$, vertical arrows). There were no significant differences in the mean cycle period across joints at any stimulation frequency or for any joint across stimulation frequencies, except for a shorter cycle period of the knee at $10 \mathrm{~Hz}$ and of the hip at $20 \mathrm{~Hz}$ compared with $1 \mathrm{~Hz}$ (Fig. 2B). The amplitude of the knee joint movements increased progressively from 1 to $10 \mathrm{~Hz}$ : the amplitudes were higher at 5, 10, and $20 \mathrm{~Hz}$ than at $1 \mathrm{~Hz}$ and higher at 10 and $20 \mathrm{~Hz}$ than at $5 \mathrm{~Hz}$ (Fig. 2C). The mean amplitudes of the hip and ankle were not significantly different across frequencies. Consistent ankle joint movements were observed only during stimulation at 5 and $10 \mathrm{~Hz}$ (Fig. $2 \mathrm{~B}, \mathrm{C}$ ).

The effects of a $10 \mathrm{~s}$ train of SEMS ( $60 \%$ of maximum tesla at T11-T12 vertebral level) at 1 and $3 \mathrm{~Hz}$ on the organization of reflex activity in the leg muscles were compared (Fig. 3). SEMS at $1 \mathrm{~Hz}$ evoked responses to each stimulus in the RF and BF (Fig. $3 A, C)$, and the superposition of 10 traces demonstrates that these responses were time linked to the stimulus (Fig. $3 E$ ). Unlike at 1 $\mathrm{Hz}$, SEMS at $3 \mathrm{~Hz}$ induced a sustained locomotor-like pattern of EMG bursting activity (Fig. 3, compare $A, B$ ). In three of seven subjects, when stimulating at $1 \mathrm{~Hz}$ there was a latency of $\sim 70$ $150 \mathrm{~ms}$ for the first EMG response to appear with the extensor burst having a slightly longer latency than the flexor burst (Fig.
$\mathrm{T} 11-\mathrm{T} 12$
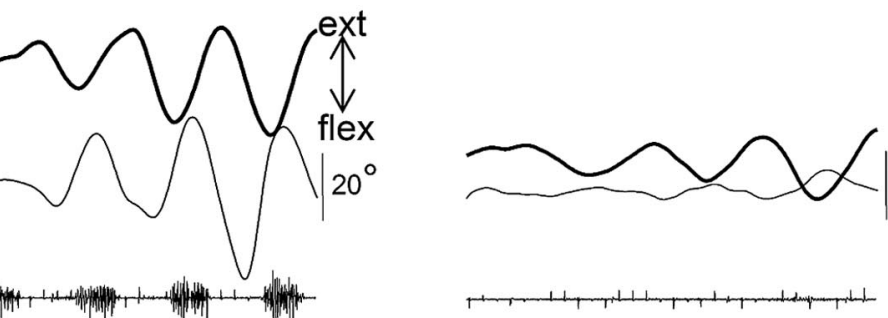

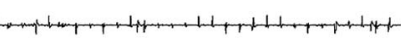

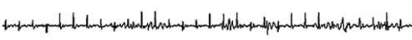

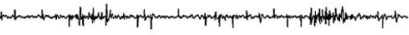
$0.2 \mathrm{mV}$

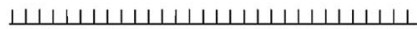

$2 \mathrm{sec}$
L1-L2

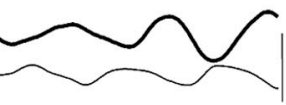

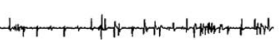

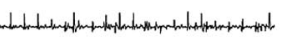

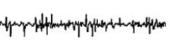

1111111111

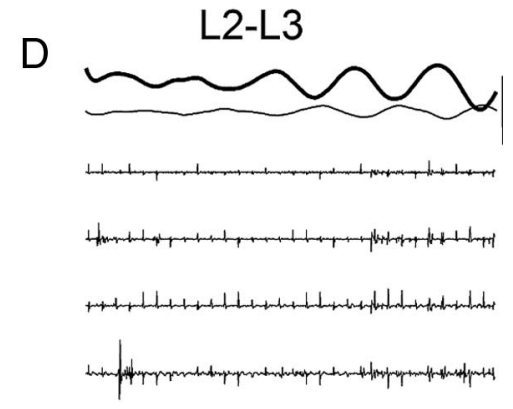

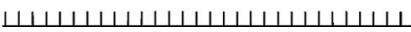

Figure 1. $\quad \boldsymbol{A}-\boldsymbol{D}$, Angular movements of right hip $[\mathrm{Hip}(\mathrm{R})]$ and right knee $[K n e e(\mathrm{R})]$ joints as well as representative EMG activity

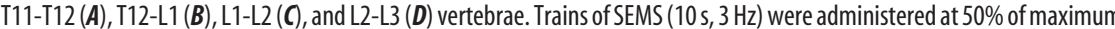
BF when SEMS was applied at T11-T12, but minimal oscillations were observed at the other spinal levels. Upward and downward deflections of the hip and knee joint angle traces denote extension (ext) and flexion (flex), respectively.

$3 E)$. Even within a subject, the exact delay of the flexor and extensor responses varied between individual stimuli. Subsequently, there is little or no EMG response before the next stimulus (Fig. 3C). At $3 \mathrm{~Hz}$ the initial EMG response has a latency of $\sim 80 \mathrm{~ms}$ to the first stimulus and is followed by a more prolonged burst of activity as a result of the shorter interval between the first and second stimulus (Fig. 3D). It appears that stimulation at $1 \mathrm{~Hz}$ is insufficient to initiate a level of excitability to sustain a rhythmic bursting pattern, whereas the stimulus interval at $3 \mathrm{~Hz}$ can progressively enhance the excitability of the circuitry so that rhythmic and coordinated bursting can be sustained. Superposition of 30 traces at $3 \mathrm{~Hz}$ showed that the EMG responses were not closely time linked to the stimulus (Fig. $3 F$ ), i.e., there was no consistent latency in the response to individual stimuli. Thus, we conclude that SEMS at $3 \mathrm{~Hz}$ can activate at least some components of the spinal neuronal locomotor networks and initiate step-like movements in normal individuals.

\section{Strength of SEMS}

We compared the effects of SEMS strength ranging from 40 to $70 \%$ of maximum during constant frequency stimulation $(3 \mathrm{~Hz}$ at T11-T12 vertebral level). SEMS at 40\% strength induced some rhythmic, low-amplitude movements at the hip and knee joints (Fig. $4 A, C$ ) and low-amplitude, sporadic EMG activity in the BF and RF muscles (Fig. 4A). With SEMS at 50, 60, or 70\% strength, the hip and knee moved in opposite directions rhythmically: the 

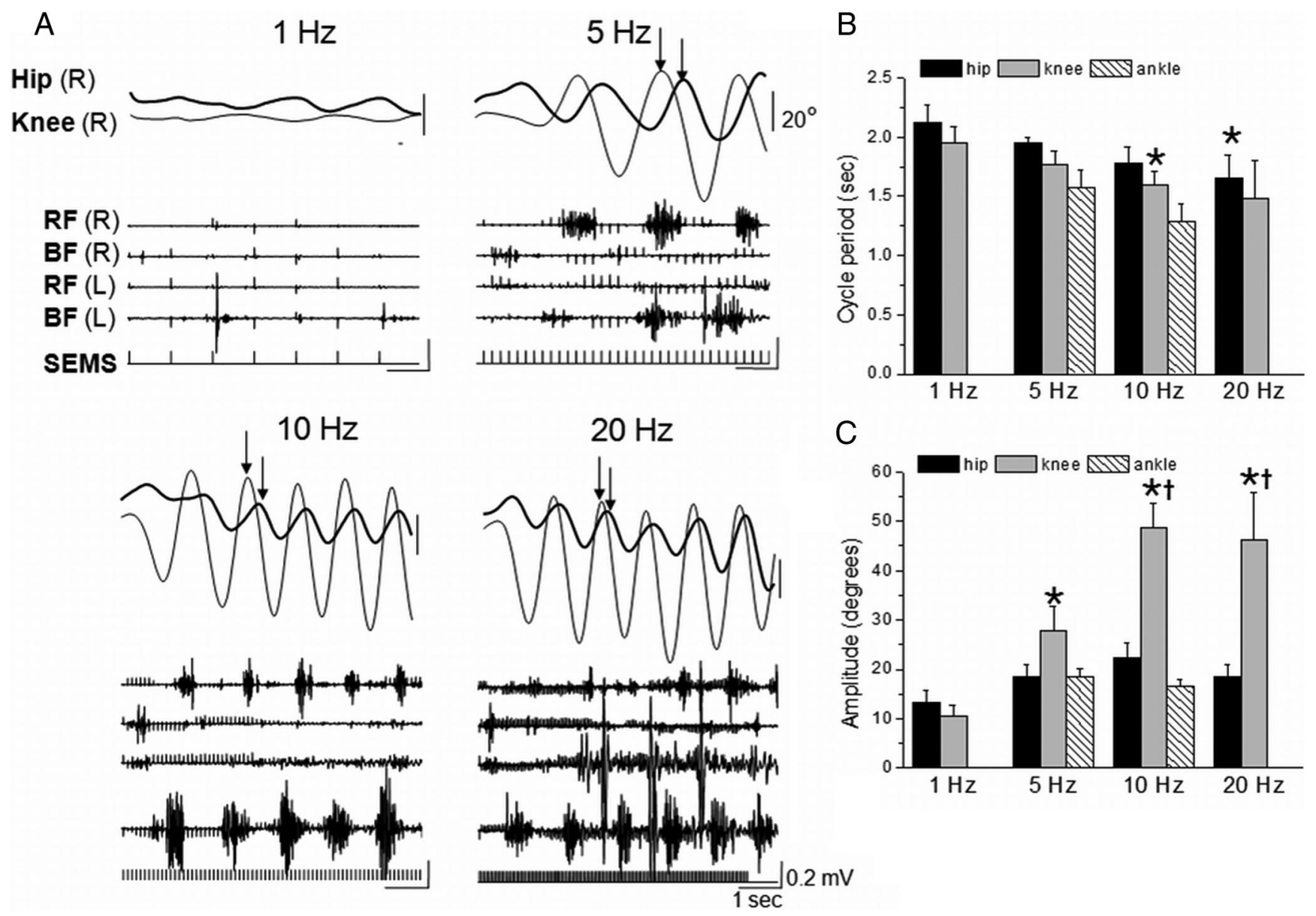

Figure 2. Effects of different frequencies of SEMS over the T11-T12 vertebrae on involuntary locomotor-like stepping movements. $A$, Angular cyclic movements in the hip (R) and knee (R) joints and the EMG activity in the RF (R), BF (R), RF (L), and BF (L) during SEMS at 1,5, 10, and $20 \mathrm{~Hz}$ are shown. Arrows denote the phase shifts for the hip and knee joint excursions. $B, C$, The duration of the cycle period $(\boldsymbol{B})$ and amplitude $(\boldsymbol{C})$ of the hip, knee, and ankle movements during SEMS at each frequency are shown. Values are means \pm SE for seven subjects, but ankle movements were observed only at 5 and $10 \mathrm{~Hz}$ in four subjects. * and $\dagger$, Significantly different from $1 \mathrm{~Hz}$ and $5 \mathrm{~Hz}$, respectively, at $p<0.05$. Abbreviations are the same as those used in Figure 1.

hip joint extended and the knee joint flexed with an $\sim 0.7$, $\sim 0.6$, or $\sim 0.6$ s delay between knee and hip flexion, respectively (Fig. $4 A$, arrows). The cycle period of the hip was progressively shorter from 40 to $70 \%$ stimulus strength: the mean cycle periods at 50,60 , and $70 \%$ were shorter than at $40 \%$, and the mean cycle period at $70 \%$ was shorter than at $50 \%$ (Fig. $4 B$ ). No significant differences in cycle period were observed across strengths for the knee and ankle, except that the mean cycle period was shorter at $70 \%$ than at $40 \%$ for the knee. The amplitudes of the movements of the hip at $70 \%$ were greater than at 40 and $50 \%$ (Fig. $4 C$ ). The mean amplitudes for the knee were significantly higher with each increase in stimulation strength. SEMS at 50-70\% also initiated rhythmic movements at the ankle joint in four of the subjects, and there were no differences in mean cycle periods (Fig. $4 B$ ) or amplitudes (Fig. 4C) across stimulation strengths.

\section{Effects of vibration plus SEMS}

Figure 5 demonstrates the effects of the addition of SEMS to vibration in two subjects. In one subject, vibration alone did not induce leg movements (Fig. 5A). The addition of SEMS, however, immediately elicited locomotor-like movements accompanied by rhythmic EMG bursting activity in the leg muscles. All joint movements began after the onset of SEMS and continued after SEMS was terminated. In another subject (Fig. $5 B$ ), vibration induced small-amplitude locomotor-like movements at all joints and some EMG activity in the leg muscles. Addition of SEMS dramatically facilitated the locomotor-like activity and EMG amplitudes. After SEMS was terminated but vibration continued, the locomotor-like activity remained potentiated relative to the activity induced by the initial phase of vibration alone in both subjects (Fig. $5 A, B$ ).

\section{EMG and kinematics features of the locomotor-like activity} induced under each condition

The locomotor-like activity in response to each of the stimulation conditions (SEMS, vibration, vibration plus SEMS, and voluntary air stepping) for the same subject during the same test session is shown in Figures 6 and 7. These responses were generally representative across all seven subjects. EMG bursts during SEMS were present in more muscles than was observed during vibration, but the combination of vibration plus SEMS resulted in the most robust bursting with the highest amplitudes (Fig. 6A). Note that during vibration there was a gradual increase in tonic activity that subsequently transformed into a bursting activity at an average latency of $2.45 \pm 0.28 \mathrm{~s}$ (mean for five subjects). In contrast, the latency for the bursting activity in response to SEMS and vibration plus SEMS was only $0.68 \pm 0.10$ and $0.47 \pm 0.10 \mathrm{~s}$, respectively (mean for seven subjects). The movements of the lower limb illustrated by stick figures and the trajectories of the 
hallux were qualitatively similar across conditions, but in response to SEMS the hallux trajectory reflected less flexion during the swing phase of the step cycle than under all other conditions (Fig. 6B). In contrast, the greatest degree of limb flexion was observed during voluntary stepping.

The coordination of the hip, knee, and ankle was qualitatively similar across all conditions, with the peak flexion occurring first at the knee, then the hip, and then the ankle (Fig. 7A). The coordination between the knee and hip and between the ankle and knee are also qualitatively similar as can be seen by the interjoint projections shown in Figure $7 \mathrm{~B}$. In two subjects, bilateral stepping movements in the right and left knee joints were recorded using goniometers during SEMS and vibration plus SEMS showing alternating flexion and extension between the two legs (supplemental Figs. 1, 2 , available at www.jneurosci.org as supplemental material).

In normal locomotion the cycle periods as determined by the kinematics of the hip, knee, or ankle are identical. This tight linking of the three joints was not always present in response to SEMS or vibration (Fig. 8A). For example, in response to SEMS or vibration the mean cycle periods as determined at the knee and ankle were shorter than at the hip, reflecting that occasionally an extra cycle was generated at the knee and ankle relative to the hip. In addition, the cycle period for the ankle during vibration plus SEMS was shorter than that for the hip. The mean cycle periods for the hip during vibration plus SEMS and voluntary stepping were shorter than during SEMS or vibration alone. For the knee, the mean cycle periods during vibration plus SEMS and voluntary stepping were shorter than during SEMS alone. For the ankle, the mean cycle period during vibration plus SEMS was shorter than during SEMS alone. When vibration and SEMS were combined, the amplitude of the hip displacement was greater than with vibration alone (Fig. $8 \mathrm{~B}$ ). The amplitude at the hip was greater during voluntary air stepping than during each of the other conditions. The amplitudes at the knee were larger during vibration plus SEMS than during either condition alone. Knee displacement was even higher during voluntary air stepping. No differences were observed in the amplitude at the ankle among the four conditions.

\section{Discussion}

\section{Significance of a general command capability (automaticity) for locomotion}

Four decades ago Shik et al. (1966) reported that stepping could be induced in decerebrated cats using a simple tonic stimulation pattern applied to the mesencephalic locomotor nucleus. Those observations are consistent with the concept that the role of supraspinal control of stepping could be minimized in its complexity by a simple general command "to walk." The present observations are novel in demonstrating that this level of general control also exists in uninjured humans and that command signals can be initiated at the lumbosacral spinal cord level.
Given this general command capability, the importance of the present observations is threefold. First, present results confirm previous observations that the lumbosacral spinal cord of noninjured humans contains the circuitry that can induce stepping-like movements by mechanical (vibration) (Gurfinkel et al., 1998; Isaev et al., 2004) or tonic electrical stimulation via sensory input to the spinal cord (Selionov et al., 2009). The results also demonstrate that the more direct stimulation of the spinal cord locomotor circuitry by SEMS can initiate and sustain movements more robustly than by stimulation of sensory input (via muscle vibration) to this circuitry. Second, the results demonstrate the feasibility of accessing the lumbosacral locomotor circuitry using a noninvasive, pain-free procedure. Third, this study provides the first example of using SEMS to induce involuntary, complex, coordinated behaviors that are relatively automatic in nature.

\section{Evidence for indirect versus direct access to the spinal locomotor circuitry: initiation of cyclic locomotor activity by afferent stimulation}

Gurfinkel et al. (1998) showed that in $\sim 50 \%$ of noninjured human subjects tested, involuntary locomotor-like movements could be induced by tonic vibration of the quadriceps in one leg when subjects were lying on their side and their legs suspended in a gravity-neutral position. Presumably, the predominant vibration-related sensory information generated was via activation of muscle spindles and Ia fibers, with the likelihood of activation of some Ib fibers and skin receptors.

A second approach for modulating sensory input was tonically stimulating the peroneal or sural nerve (Selionov et al., 2009). Sensory nerve electrical stimulation initiates stepping 
A $\quad 40 \%$
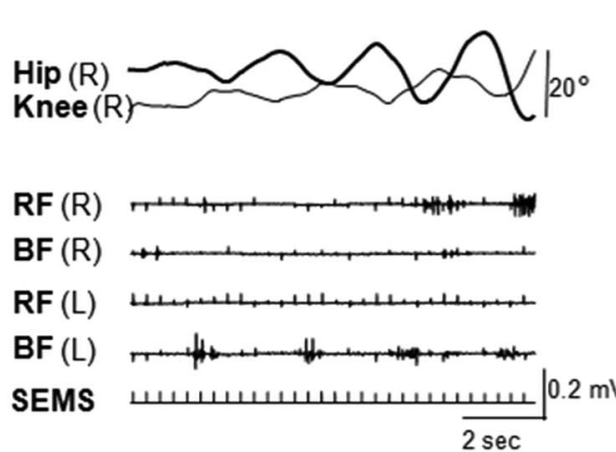

$60 \%$
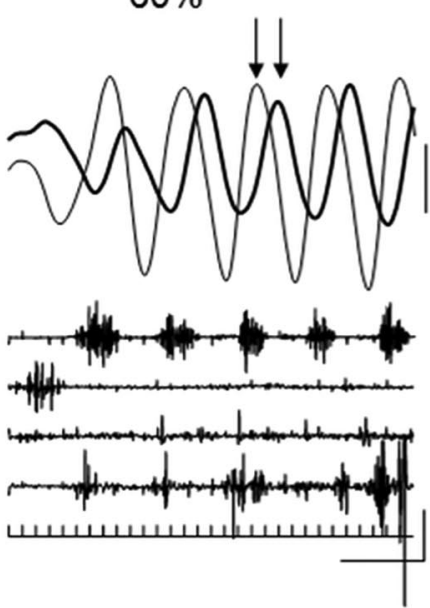
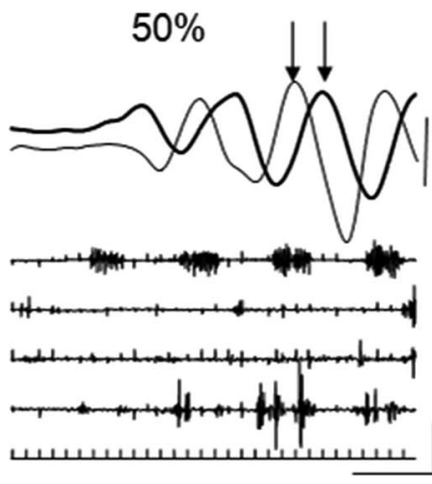

$70 \%$

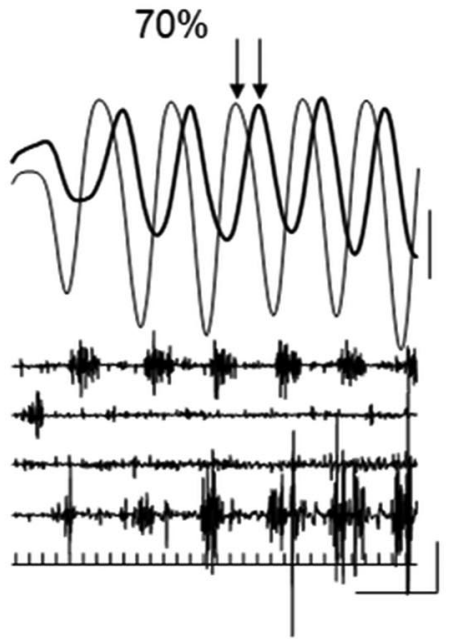

B

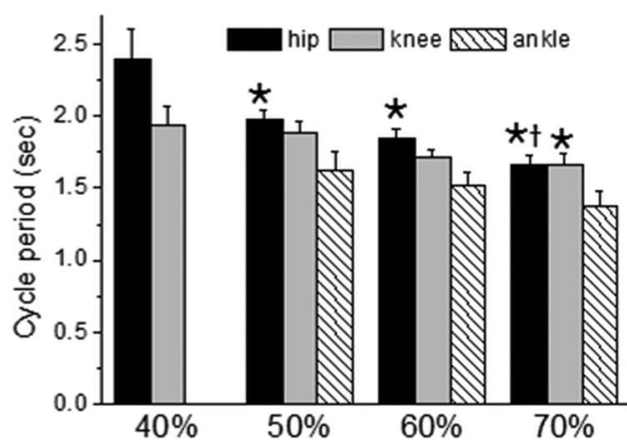

C

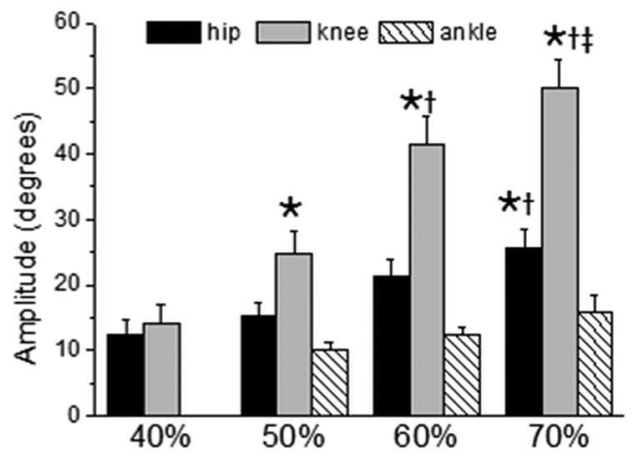

Figure 4. A, Kinematics for the hip (R) and knee (R) and the EMG patterns for the RF ( $R$ and $\mathrm{L}$ ) and $\mathrm{BF}$ ( $\mathrm{R}$ and $\mathrm{L}$ ) during SEMS ( $3 \mathrm{~Hz}$ at $\mathrm{T} 11-\mathrm{T} 12$ ) at $40,50,60$, and $70 \%$ of maximum tesla. Arrows at 50,60 , and $70 \%$ denote the phase shift between the hip and knee joint excursions. $\boldsymbol{B}, \boldsymbol{C}$, The durations of the cycle periods $(\boldsymbol{B})$ and amplitudes $(\boldsymbol{C})$ of the hip, knee, and ankle movements for each strength of SEMS are shown. Values are means $\pm S E .{ }^{*}, \dagger$, and $\neq$, Significantly different from 40,50, and $60 \mathrm{~Hz}$, respectively, at $p<0.05$. Abbreviations are the same as those in Figure 1.

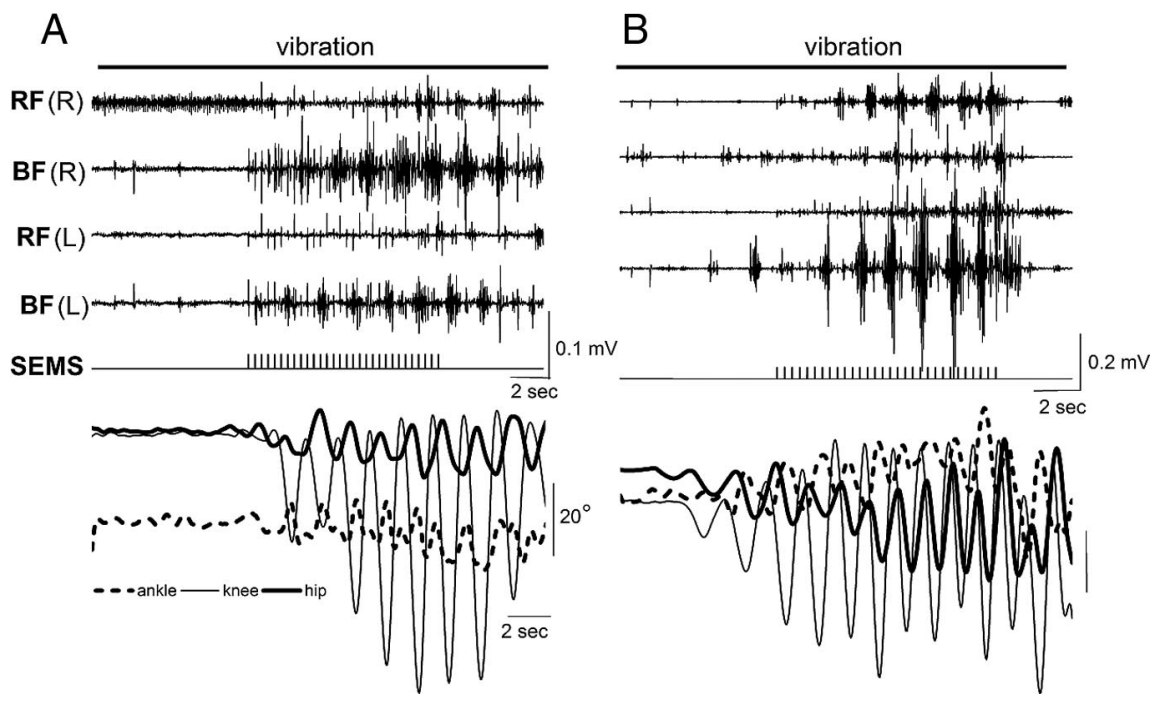

Figure 5. $A, B$, The effects of the addition of SEMS to vibration of the leg muscles. One subject showed no rhythmic response to vibration $(\sim 1 \mathrm{~mm}, 50 \mathrm{~Hz}$ ) alone $(\boldsymbol{A})$, whereas a second subject showed some rhythmic activity (B). Addition of SEMS ( $3 \mathrm{~Hz}$ at T11-T12 at $60 \%$ of maximum tesla) to vibration in the subject shown in $\boldsymbol{A}$ initiated locomotor-like activity at the hip, knee, and ankle joints and rhythmic EMG activity in the RF and BF bilaterally. Addition of SEMS to vibration in the subject shown in $B$ enhanced the ongoing locomotor-like activity and EMG bursting elicited by vibration alone. Abbreviations are the same as those used in Figure 1. movements with an $\sim 5$ s delay (Selionov et al., 2009). This is approximately the same time necessary for muscle mechanical vibration to initiate rhythmic leg movements (present data) (Gurfinkel et al., 1998). Similarly, we have shown that epidural stimulation $(5 \mathrm{~Hz})$ at the $\mathrm{L} 5 \mathrm{spi}$ nal level in decerebrated cats initially induces tonic activity in hindlimb muscles that transforms to locomotor-like activity after 5-7 s of stimulation (Gerasimenko et al., 2005). These observations are consistent with some indirect, delayed access of sensory systems to the spinal locomotor circuitry. In contrast, cyclic locomotorlike activity induced by SEMS was initiated almost immediately after the first stimulus without any preceding tonic phase, suggesting a direct activation of the spinal locomotor circuitry.

The above observations suggest that the locomotor circuitry is located within spinal and/or supraspinal circuits, with either or both capable of inducing cyclic motor output (Shik, 1997). Tonic stimulation via chronically implanted epidural 
A
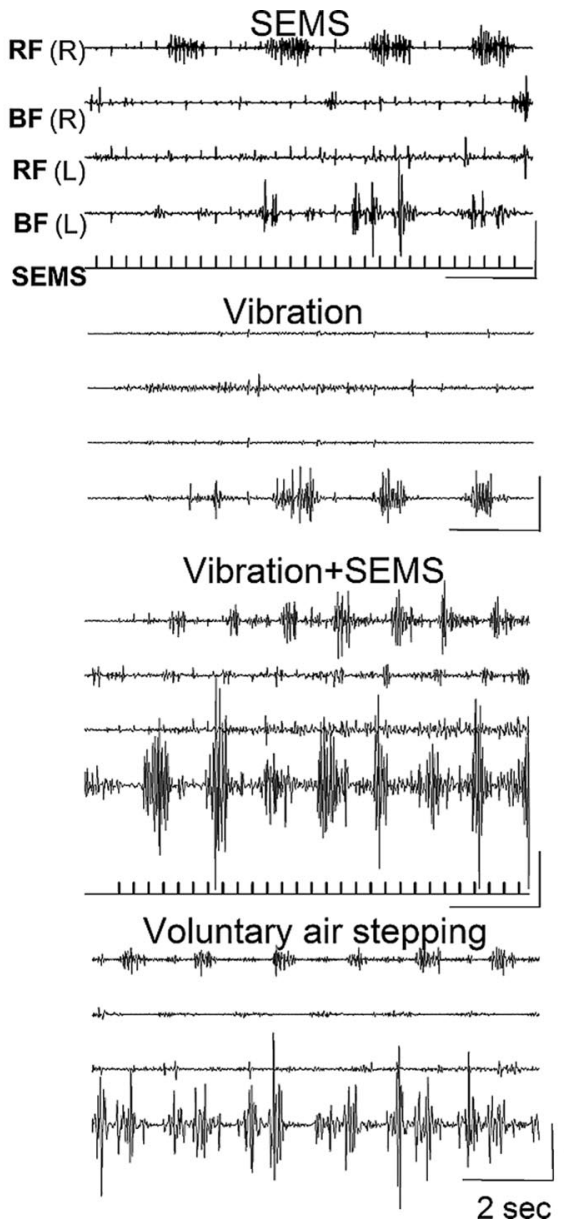

B

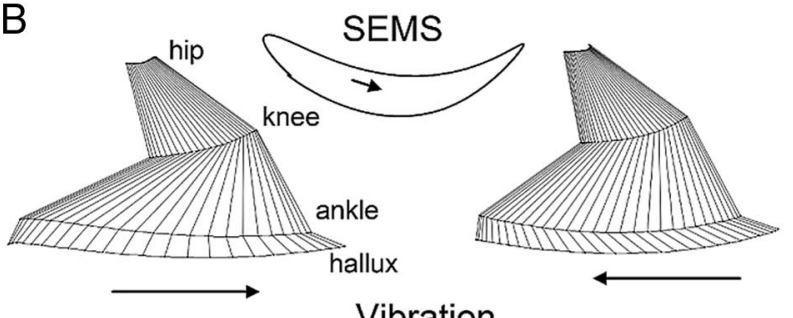

Vibration

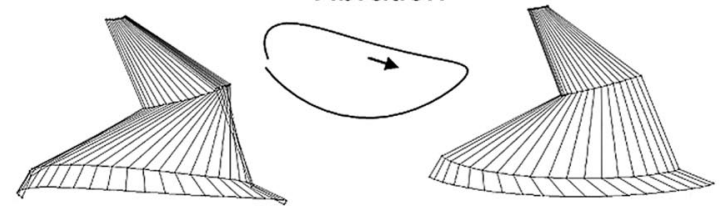

Vibration+SEMS
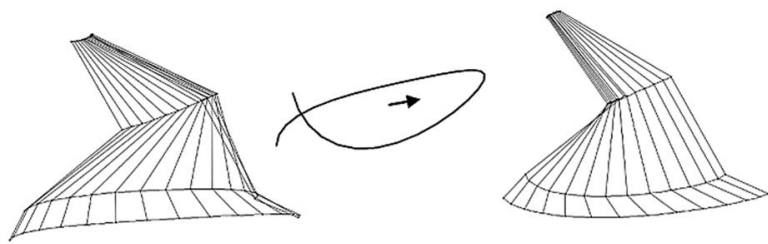

Voluntary air stepping
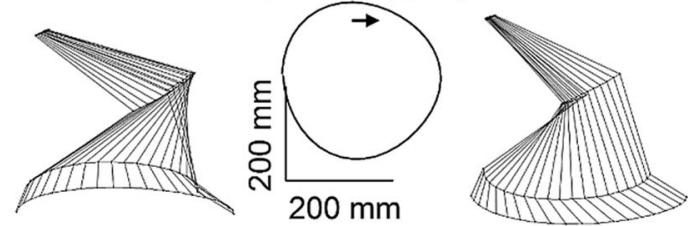

Figure 6. $\quad \boldsymbol{A}, \boldsymbol{B}, \mathrm{EMG}$ and kinematic features of locomotor patterns induced under each experimental condition. EMG patterns in the $\mathrm{RF}(\mathrm{R}$ and $\mathrm{L}$ ) and $\mathrm{BF}$ (R and $\mathrm{L}$ ) muscles under each experimental condition are shown in $\boldsymbol{A}$. Stick diagram decompositions ( $40 \mathrm{~ms}$ between sticks) of the movements of the right leg during one step cycle are displayed in $\boldsymbol{B}$. Also shown in $\boldsymbol{B}$ are the trajectories of the limb endpoint (hallux marker) for one step cycle. Arrows in $\boldsymbol{B}$ indicate the direction of movement. Abbreviations are the same as those used in Figure 1.

electrodes can induce stepping-like movements in humans with a complete spinal cord injury when lying in a supine position (Dimitrijevic et al., 1998; Shapkova, 2004; Minassian et al., 2007; Gerasimenko et al., 2008). We have shown that robust full weight-bearing stepping can be generated via epidural stimulation in chronic, complete spinal, step-trained rats (Courtine et al., 2009). The mechanism for this effect of epidural stimulation in humans has been attributed to dorsal root afferents (Minassian et al., 2007) that could activate the cyclic locomotor circuitry. Theoretically, the basic mechanism for accessing the spinal circuitry to induce these movements via epidural stimulation appears to be indirect, similar to peripheral nerve stimulation and muscle vibration. Minassian et al. (2007) reported that each epidurally induced pulse during an EMG burst elicited a separate reflex response (termed posterior root reflex) in spinal cordinjured subjects and suggested that this tonic input (via sensory axons) activates a locomotor interneuronal network.

In addition to differences in the time course and emergence from a tonic to a phasic pattern of stepping in response to sensory stimulation vs SEMS, other features of the patterns of evoked EMG responses to SEMS are consistent with a more direct activation of the spinal locomotor circuitry. There were no strictly time-linked evoked responses within the rhythmic EMG bursts associated with SEMS at $3 \mathrm{~Hz}$. In response to SEMS, we observed triggered responses with an $\sim 80-100 \mathrm{~ms}$ latency that initiated EMG bursting activity in both flexor (BF) and extensor (RF) muscles (Fig. $3 B, D)$. There was a much shorter $(\sim 10-15 \mathrm{~ms})$ and consistent latency for evoked reflex responses within EMG bursts of the quadriceps and hamstrings with epidural stimulation at L2 in spinal cord-injured subjects (Minassian et al., 2004). Epidurally evoked responses also were phase-dependently modulated, suggesting that pathways mediating these fixed shortlatency responses are part of the circuitry that generates cyclic motor activity.

Another fundamental difference in the mechanisms of activation of locomotor movements with SEMS vs epidural stimulation is stimulation frequency. In the present study, SEMS as low as 3 $\mathrm{Hz}$ induced locomotor-like movements in the legs of noninjured subjects. In contrast, epidural stimulation at low frequencies did not elicit any stepping-like patterns in individuals with a complete spinal cord injury (Jilge et al., 2004; Minassian et al., 2007), with the optimal frequency being $\sim 40 \mathrm{~Hz}$ (Dimitrijevic et al., 1998). This marked difference in stimulation frequency provides further evidence that the more superficial dorsal root afferents are activated with epidural stimulation compared with a more direct stimulation of the spinal locomotor circuitry consisting of complex networks of interneurons with SEMS. With the available resources, we could not determine the effects of a wider range of SEMS frequencies. Future studies will be needed to explain further the mechanistic differences in stimulation frequency responses between SEMS and epidural stimulation. 

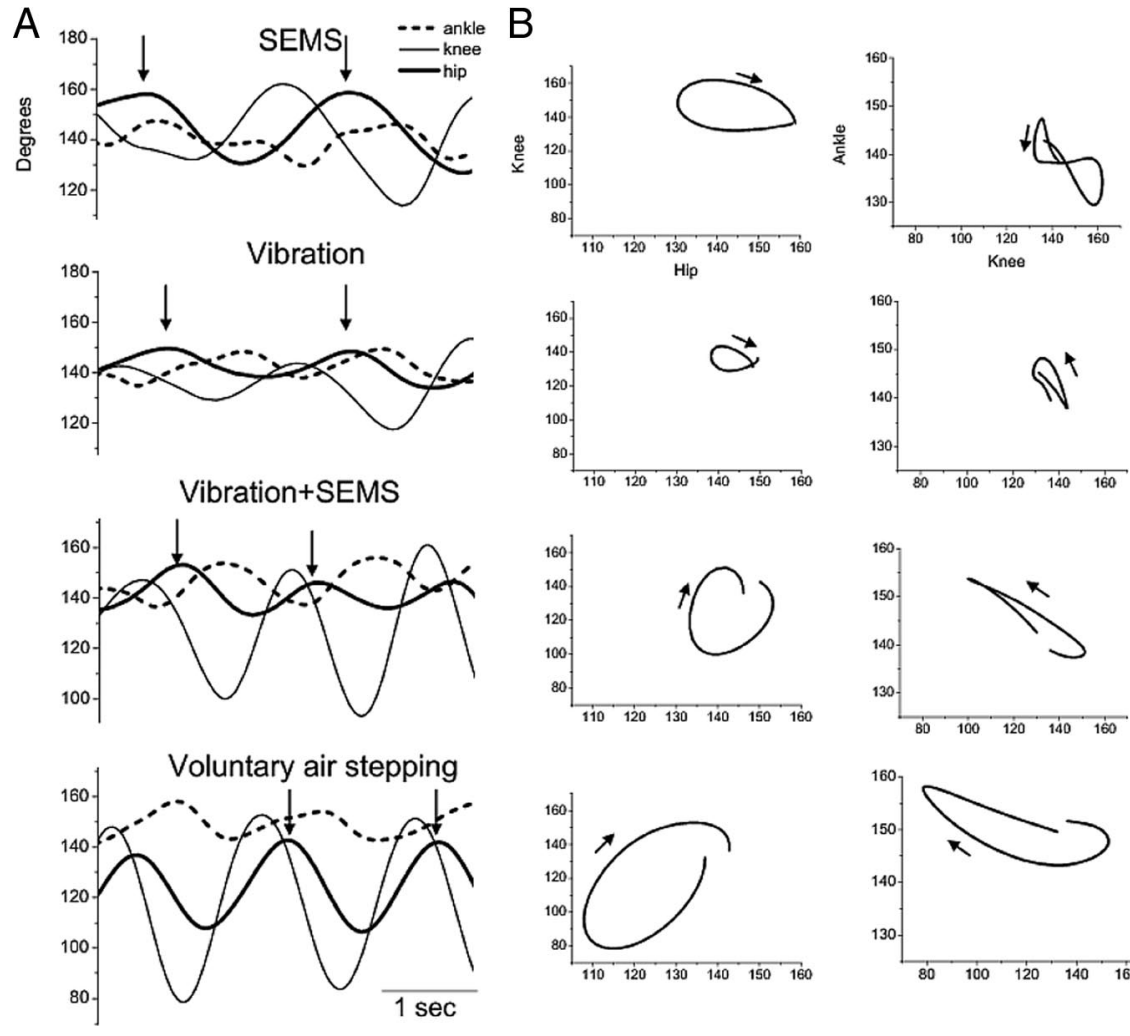

Figure 7. $\quad \boldsymbol{A}, \boldsymbol{B}$, Interjoint coordination under each experimental condition. Joint angle plots of flexion (downward movement) and extension (upward movement) of the hip, knee, and ankle are illustrated for each experimental condition. Angle-angle plots showing the coupling between the hip and knee (left) and knee and ankle (right) for one step cycle are shown in $\boldsymbol{B}$. Arrows in $\boldsymbol{A}$ mark the initiation and termination within the step cycle that is illustrated in $\boldsymbol{B}$. Arrows in $\boldsymbol{B}$ indicate the direction of movement. Abbreviations are the same as those used in Figure 1.
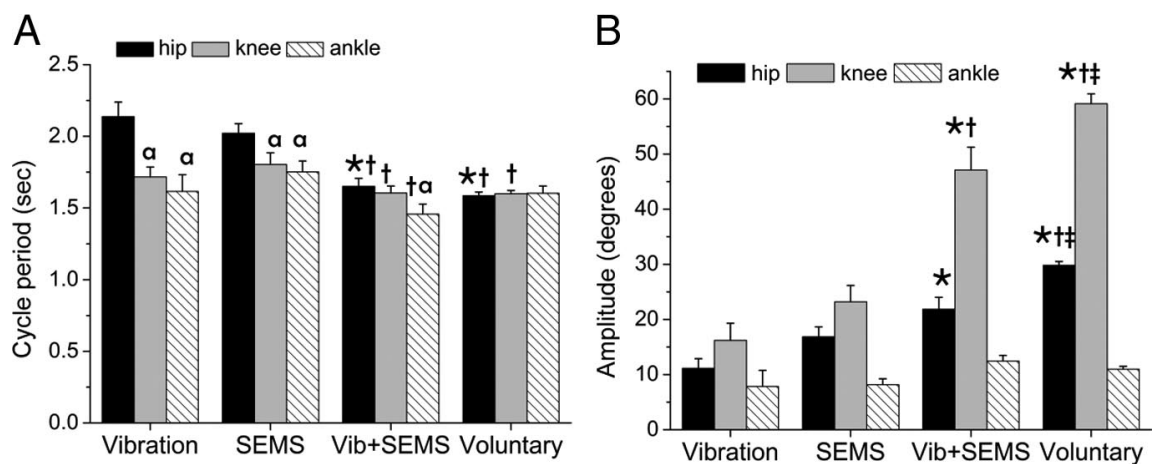

Figure 8. $\boldsymbol{A}, \boldsymbol{B}$, Durations of the cycle periods for the hip, knee, and ankle movements $(\boldsymbol{A})$ and amplitudes of these movements (B) during SEMS ( $3 \mathrm{~Hz}$ at T11-T12 at $60 \%$ of maximum tesla) alone, Vibration ( $\sim 1 \mathrm{~mm}, 50 \mathrm{~Hz}$ ) alone, vibration plus SEMS $\left(\right.$ Vib + SEMS), and voluntary air stepping. Values are means \pm SE for seven subjects, except for vibration alone $(n=5)$. ${ }^{*}, \dagger$, and $\ddagger$, Significantly different from SEMS, vibration, and vibration plus SEMS for individual joints, respectively; a, significantly different from the hip within a condition; $p<0.05$.

\section{Significance of combining vibration and SEMS}

The complementary effects of vibration and SEMS leave the possibility that both stimuli could be mediated via similar or separate mechanisms. For example, hip movement amplitudes and velocities are greater with vibration and SEMS combined compared with either alone (Fig. 8A,B). The higher movement amplitudes and velocities can be explained by a higher proportion of motor units being recruited within each relevant motor pool for vibration and SEMS combined. Theoretically, this could result from greater excitation of first-order interneurons projecting to rel-
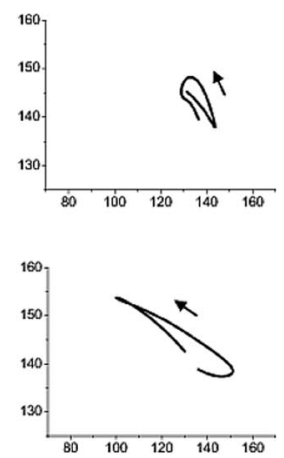

evant motor pools. The end result is that the combined sources of stimulation modulated the pattern, but not rhythm properties, of the locomotor circuitry. An underlying assumption of this interpretation is that indirect stimulation of the locomotor circuitry via afferents by vibration and more direct stimulation by SEMS were mediated by the same functional pools of interneurons and motoneurons. Intuitively, it seems unlikely that there would be a separate set of first-order interneurons activated by sensory afferents and by SEMS, both having access to cyclic generating circuitries that would project to the relevant motor pools.

\section{Significance of the present observations to central pattern generation}

While the present experiments were not designed to address the issue of the presence of central pattern generation in the human spinal cord, this issue is inevitably raised in studies of human locomotion. We cannot discount the contribution of sensory afferents or modulation via brainstem circuits and, therefore, cannot attribute our observations to central pattern generation. Based on our placebo testing procedures, it is almost certain that movements were not generated consciously, i.e., cortically mediated. From a perspective of understanding the properties of the functional locomotor circuitry, the evidence is incontrovertible that any type of functional control of locomotion in the absence of supraspinal input must include sensory control (Edgerton et al., 2008; Grillner et al., 2008). This is not to say that cyclic motor outputs cannot be generated without sensory input to networks capable of central pattern generation, but this cyclic output in itself cannot generate functional locomotion. For example, this cyclic motor output does not have the ability to adjust itself to successfully cope with constantly changing external conditions associated with weightbearing stepping. We propose that a critical issue that has received little attention to date is how complex patterns of sensory input associated with weightbearing stepping are processed by a spinal circuitry having central pattern generation capability (Courtine et al., 2009).

\section{Clinical application}

An important question is whether SEMS can provide sufficient excitability of the spinal circuitry caudal to a severe spinal cord injury to facilitate load-bearing locomotion. Another critical question is whether the locomotor-like patterns observed can occur in the absence of supraspinal connectivity, i.e., support from brainstem circuitries. A third critical question is whether 
weeks or months after a spinal cord injury the spinal interneurons have been rendered sufficiently nonfunctional to be unable to produce the necessary coordination mediated by spinal interneurons that project to those motor pools that generate stepping. Each of these questions needs to be studied carefully to determine the potential of SEMS as an intervention tool to facilitate recovery of stepping after a severe spinal cord injury.

\section{References}

Courtine G, Harkema SJ, Dy CJ, Gerasimenko YP, Dyhre-Poulsen P (2007) Modulation of multisegmental monosynaptic responses in a variety of leg muscles during walking and running in humans. J Physiol 582:11251139.

Courtine G, Gerasimenko Y, van den Brand R, Yew A, Musienko P, Zhong H, Song B, Ao Y, Ichyama R, Lavrov I, Roy RR, Sofroniew MV, Edgerton VR (2009) Transformation of nonfunctional spinal circuits into functional states after the loss of brain input. Nat Neurosci 12:1333-1342.

Di Lazzaro V, Oliviero A, Pilato F, Saturno E, Dileone M, Mazzone P, Insola A, Tonali PA, Rothwell JC (2004) The physiological basis of transcranial motor cortex stimulation in conscious humans. Clin Neurophysiol 115:255-266.

Dimitrijevic M, Gerasimenko Yu, Pinter M (1998) Evidence for a spinal central pattern generator in humans. Ann N Y Acad Sci 860:360-376.

Edgerton VR, Courtine G, Gerasimenko YP, Lavrov I, Ichiyama RM, Fong AJ, Cai LL, Otoshi CK, Tillakaratne NJ, Burdick JW, Roy RR (2008) Training locomotor networks. Brain Res Rev 57:241-254.

Fitzgerald P, Fountain S, Daskalakis Z (2006) A comprehensive review of the effects of rTMS on motor cortical excitability and inhibition. Clin Neurophysiol 117:2584-2596.

Gerasimenko Y, Lavrov I, Bogacheva I, Sherbakova N, Kucher V, Musienko P (2005) Formation of locomotor patterns in decerebrated cats in conditions of epidural stimulation of the spinal cord. Neurosci Behav Physiol 35:291-298.

Gerasimenko Y, Roy RR, Edgerton VR (2008) Epidural stimuiation: comparision of spinal circuits that generate and control locomotion in rats, cats and humans. Exp Neurol 209:417-425.

Gerasimenko Y, Musienko P, Bogacheva I, Moshonkina T, Savochin A, Lavrov I, Roy RR, Edgerton VR (2009a) Propriospinal bypass of the serotonergic system that can facilitate stepping. J Neurosci 29:5681-5689.

Gerasimenko Y, Gorodnichev R, Musienko P, Roy R, Edgerton R (2009b) Novel access to the cat and human locomotor circuitry. Soc Neurosci Abstr 35:564.2.

Gerasimenko YP, Ichiyama RM, Lavrov IA, Courtine G, Cai L, Zhong H, Roy RR, Edgerton VR (2007) Epidural spinal cord stimulation plus quipazine administration enable stepping in complete spinal adult rats. J Neurophysiol 98:2525-2536.
Grillner S, Wallén P, Saitoh K, Kozlov A, Robertson B (2008) Neural bases of goal-directed locomotion in vertebrates-an overview. Brain Res Rev $57: 2-12$.

Gurfinkel VS, Levik YS, Kazennikov OV, Selionov VA (1998) Locomotorlike movements evoked by leg muscle vibration in humans. Eur J Neurosci 10:1608-1612.

Gurfinkel VS, Levik YS, Kazennikov OV, Selionov VA (2000) Jendrassik's maneuver creates the conditions for triggering involuntary stepping movements (in Russian). Fiziol Cheloveka 26:73-79.

Hallett M (2000) Transcranial magnetic stimulation and the human brain. Nature 406: 147-150.

Hallett M (2007) Transcranial magnetic stimulation: a primer. Neuron 55:187-199.

Huerta PT, Volpe BT (2009) Transcranial magnetic stimulation, synaptic plasticity and network oscillations. J Neuroeng Rehabil 6:7.

Ichiyama RM, Gerasimenko YP, Zhong H, Roy RR, Edgerton VR (2005) Hindlimb stepping movements in complete spinal rats induced by epidural spinal cord stimulation. Neurosci Lett 383:339-344.

Isaev G, Gerasimenko Y, Selionov V, Kartashova N (2004) Respiratory responses to voluntary and reflexly induced stepping movements in normal subjects and spinal patients. J Physiol Pharmacol 55:77-82.

Jilge B, Minassian K, Rattay F, Pinter MM, Gerstenbrand F, Binder H, Dimitrijevic MR (2004) Initiating extension of the lower limbs in subjects with complete spinal cord injury by epidural lumbar cord stimulation. Exp Brain Res 154:308-326.

Lavrov I, Dy CJ, Fong AJ, Gerasimenko Y, Courtine G, Zhong H, Roy RR, Edgerton VR (2008) Epidural stimulation induced modulation of spinal locomotor networks in adult spinal rats. J Neurosci 28:6022-6029.

Minassian K, Jilge B, Rattay F, Pinter M, Binder H, Gerstenbrand F, Dimitrijevic M (2004) Stepping-like movements in humans with complete spinal cord injury induced by epidural stimulation of the lumbar cord: electromyographic study of compound muscle action potentials. Spinal Cord 42:401-416.

Minassian K, Persy I, Rattay F, Pinter M, Kern H, Dimitrijevic M (2007) Human lumbar cord circuitries can be activated by extrinsic tonic input to generate locomotor-like activity. Hum Mov Sci 26:275-295.

Selionov VA, Ivanenko YP, Solopova IA, Gurfinkel VS (2009) Tonic central and sensory stimuli facilitate involuntary air-stepping in humans. J Neurophysiol 101:2847-2858.

Shapkova E (2004) Spinal locomotor capabality revealed by electrical stimulation of the lumbar enlargement in paraplegic patients. In: Progress in motor control (Latash M and Levin M, eds), pp 253-289. Champaign, IL: Human Kinetics.

Shik M (1997) Recognizing propriospinal and reticulospinal systems of initiation of stepping. Motor Control 1:310-313.

Shik ML, Severin FV, Orlovsky GN (1966) Control of walking and running by means of electric stimulation of the midbrain. Biofizika 11:659-666. 